\title{
40 anos da Pós-graduação da EEFE-USP: a sua contribuição para o avanço do conhecimento sobre o comportamento motor humano
}

http://dx.doi.org/10.11606/1807-55092017000nesp097

\author{
Umberto Cesar CORRÊA* \\ Jorge Alberto de OLIVEIRA* \\ Go TANI* \\ *Escola de Educação \\ Física e Esporte, \\ Universidade de São \\ Paulo, São Paulo, SP, \\ Brasil.
}

\section{Considerações iniciais}

A pós-graduação é um sistema de ensino de longa existência, principalmente em universidades europeias (e.g. Universidade de Bolonha, Universidade de Oxford e Universidade de Coimbra); no Brasil, ela é recente. $\mathrm{Na}$ Universidade de São Paulo (USP), por exemplo, os programas de pós-graduação mais antigos, dentre os quais se encontra o da Escola de Educação Física e Esporte (EEFE), datam da década de 1970.

Pode-se dizer que a pós-graduação em Educação Física no Brasil resultou da conjunção de três importantes eventos ocorridos em meados da referida década: a) a reforma universitária impulsionada pelo governo federal, que implicou em mudanças na estrutura das universidades como, por exemplo, a extinçáo da cátedra, a implantação da estrutura departamental, a instituição do regime de trabalho de dedicação integral à docência e à pesquisa (RDIDP) com destaque para a valorização da pesquisa ${ }^{1-2} ; b$ ) a influência do movimento disciplinar da Educação Física, iniciado nos EUA, cuja meta era dar à área um caráter acadêmico ${ }^{3-4}$; e c) o retorno às universidades brasileiras de docentes e pesquisadores com doutorados obtidos em universidades estrangeiras ${ }^{5-6}$.

A implantação da pós-graduação implicou, naturalmente, no crescimento da pesquisa. Esse processo, fortemente influenciado pelo paradigma científico reducionista prevalecente à época, deu origem a uma crescente especialização de áreas de pesquisa (formação de subáreas) com base no nível de análise adotado (e.g. bioquímico, fisiológico, comportamental, psicológico, sociocultural). A subárea denominada de Comportamento Motor surge no bojo dessas transformações ocorridas na universidade ${ }^{5}$. Ela passou a ser desenvolvida, basicamente, na década de 1980, mais especificamente pelas iniciativas dos professores Jefferson Tadeu Canfield, Ricardo Demétrio de Souza Petersen, Ana Maria Pellegrini e Go Tani, sendo esses dois últimos docentes da Escola de Educação Física e Esporte da Universidade de São Paulo ${ }^{7-9}$.

A pós-graduação da EEFE-USP tem como objetivo a formação de recursos humanos qualificados - docentes, pesquisadores e profissionais - para: a) selecionar, organizar e disseminar conhecimentos sobre Educação Física e Esporte; b) produzir e publicar tais conhecimentos; c) utilizá-los para resolver problemas em diferentes contextos da intervenção profissional. Esse processo de formação se dá, fundamentalmente, por meio da inserção do aluno no mundo da ciência, isto é, um conjunto de atividades que possibilitam o acesso ao legado científico das diferentes subáreas e ao método científico para produzir novos conhecimentos. A defesa da dissertaçáo de mestrado ou da tese de doutorado constitui a etapa final desse processo.

O objetivo do presente texto é apresentar a contribuição da pós-graduação da EEFE-USP para o avanço do conhecimento na subárea de Comportamento Motor. Para tanto, faz, no primeiro tópico, uma apresentação geral da subárea de Comportamento Motor com o objetivo de mostrar o contexto em que os alunos se envolvem para adquirir conhecimentos e, no segundo tópico, apresenta uma síntese dos conhecimentos produzidos pelas teses e dissertaçôes defendidas. 


\section{Comportamento motor: da base para a formação de recursos humanos}

O movimento está no coração da existência humana. A capacidade de realizar movimentos com habilidade faz a vida possível ${ }^{10-11}$. Movimentamonos para nos alimentar, proteger, trabalhar, expressar/comunicar, locomover, dentre muitas outras açôes essenciais à vida. Entende-se, como movimentos com habilidade (i.e. habilidades motoras), os movimentos propositados, realizados sem, com pouco ou muito esforço cognitivo, mas de forma eficiente. Habilidades motoras podem ser consideradas um patrimônio humano, em razão de terem sido adquiridas, modificadas e transmitidas pelos seres humanos, ao longo de sua existência, para suprirem suas necessidades, sejam elas biológicas, psicológicas, sociais ou culturais ${ }^{12}$.

Sáo incontáveis as habilidades motoras que realizamos ao longo de nossas vidas. Contudo, não nascemos com elas "prontas". Embora possamos identificar habilidades com maior ou menor predominância genética, respectivamente, habilidades motoras filogenéticas e ontogenéticas, o importante é que elas são comportamentos adquiridos. Por exemplo, durante os primeiros anos de vida adquirimos as habilidades motoras básicas de engatinhar, andar, alcançar e pegar objetos, etc. Com o passar do tempo adquirimos mais habilidades, de usar utensílios para comer, brincar, jogar, etc. E o repertório motor torna-se cada vez mais rico com a combinação de habilidades básicas e a aquisição de habilidades específicas culturalmente configuradas. Adquirimos habilidades de escrever, tocar instrumentos musicais, além daquelas presentes no exercício, ginástica, dança, luta, esporte e jogo, ou seja, próprias da Educação Física.

A pergunta básica em Comportamento Motor é: como essas habilidades são adquiridas? Mais especificamente, por que nunca realizamos a mesma habilidade igualmente (variabilidade motora)? Como conseguimos alcançar, com consistência, o mesmo objetivo da habilidade, utilizando-se de diferentes músculos e articulaçôes (equivalência motora)? 10, 13-14 .

É de longa data que pesquisadores de várias áreas e formaçôes acadêmicas buscam respostas a essas e outras questóes derivadas - e.g. ADAMs ${ }^{15}$, CLARK e Oliveira $^{16}$, Connolly ${ }^{17}$, Schmidt $^{18}$, Thomas $^{19}$ mas, foi ao longo do último século, especificamente em meados da década de 1970, que uma área específica denominada de Comportamento Motor se estruturou e assumiu o protagonismo nessa busca. Isto resultou, entre outras coisas, na criação de um periódico específico chamado Journal of Motor Behavior e nas publicaçôes de livros seminais como "Movement behavior and motor learning"20, "The basis of motor control"21, "Mechanisms of motor skill development" 22 "Human motor behavior: an introduction" 11 e de teorias clássicas como " $A$ closedloop theory of motor learning" 23 " $A$ schema theory of discrete motor skill learning" ${ }^{24}$.

A subárea de Comportamento Motor é composta por três campos de investigação ("fields of study") denominados de Controle Motor, Aprendizagem Motora e Desenvolvimento Motor. De uma forma sintética, Controle Motor tem como objetivo compreender como as habilidades motoras são planejadas e executadas ou, mais especificamente, como: a) as informaçōes do ambiente são interpretadas; b) as decisóes são tomadas para para responder às demandas ambientais; e c) os comandos motores são organizamos para realizar tais respostas. Embora este campo tenha sido iniciado por volta de 1791, ele tem no trabalho de SHerrington ${ }^{25}$ relacionado à identificaçáo dos mecanismos básicos envolvidos no controle neural de movimentos, um dos principais influenciadores. A Aprendizagem Motora está voltada ao estudo das mudanças na capacidade de executar habilidades motoras como resultado da prática ou da experiência. Seu início é datado de 1880 com a pesquisa de BRYAN e HARTER ${ }^{26}$ sobre a aprendizagem de recepção e envio de mensagens via telégrafo. Por fim, o campo de Desenvolvimento Motor tem seu foco de estudos nas mudanças que ocorrem no movimento ao longo do ciclo de vida. O trabalho de TiedemanN ${ }^{27}$ sobre a descrição de sequências comuns e transiçôes no comportamento motor de seu filho, do nascimento aos dois anos e meio, é reconhecido como o marco inicial.

Na pós-graduação da EEFE-USP, os problemas anteriormente descritos sobre novidade, variabilidade motora, consistência motora e equivalência motora, contextualizados nos campos de investigação Aprendizagem, Controle e Desenvolvimento Motor, têm sido investigados em duas principais linhas de pesquisa: a) organização da resposta motora e aquisição de habilidades motoras; e b) análise e diagnóstico do desenvolvimento motor. A primeira tem como objetivo investigar os mecanismos e 
processos subjacentes à preparação, execução e aquisição de habilidades motoras, bem como os fatores que os influenciam. E, o objetivo da segunda é a investigação da aquisição de habilidades básicas e suas combinaçôes em crianças normais e com deficiência, e dos fatores ambientais e da tarefa que afetam o processo de desenvolvimento de indivíduos e grupos ao longo da vida.

Se, por um lado é possível distinguir os focos específicos de investigação (i.e. fenômenos) dos três campos (Aprendizagem, Controle e Desenvolvimento Motor), por outro lado é importante concebê-los como inter-relacionados, visto que os fenômenos são interdependentes, o que dá corpo à área de Comportamento Motor. De acordo com TANI $^{9}$, a aprendizagem motora implica na melhoria no controle motor, e ambos se caracterizam como mudanças que ocorrem em contexto de desenvolvimento.

Embora a subárea de Comportamento Motor seja de pesquisa básica, assume-se que a elucidação dos mecanismos e processos subjacentes à aprendizagem, ao controle e ao desenvolvimento motor possibilita uma intervenção profissional mais efetiva em contextos específicos de trabalho com habilidades motoras (ensino, treino, reabilitação) ${ }^{7,9,11,28-29}$.

\section{Das contribuições para o avanço do conhecimento}

Foram revisados 117 trabalhos, sendo 81 dissertaçóes de mestrado e 36 teses de doutorado, abordando as seguintes categorias temáticas: "feedback" ( $\mathrm{n}=5)$, prática $(\mathrm{n}=8)$, idoso ( $=10)$, instrução $(\mathrm{n}=6)$, deficiência $(\mathrm{n}=17)$, desenvolvimento $(n=15)$, cognição $(n=6)$, postura $(\mathrm{n}=5)$, transferência lateral $(\mathrm{n}=3)$, habilidades aquáticas $(\mathrm{n}=8)$, processo adaptativo $(\mathrm{n}=21)$, autocontrole $(n=6)$, atenção/visão $(n=5)$ e motivação $(n=2)$.

Como se sabe, a categorização de fenômenos não é um processo simples. Muitos dos trabalhos poderiam ser alocados em mais de uma categoria. Por exemplo, a dissertação "Efeito da frequência de conhecimento de performance na aprendizagem motora em idosos" poderia fazer parte tanto da categoria de "idosos" quanto de "feedback". Os sistemas de categorização têm sido elaborados não como um fim, mas como uma ferramenta para outros fins ${ }^{30}$. Portanto, não se pretendeu fazer da categorização uma "camisa de força" que definisse a direção de um determinado trabalho, mas, de acordo com a interpretação dos autores, algo que permitisse a organização de uma estrutura de conhecimento.

O "feedback" é uma das variáveis mais antigas estudadas na subárea de Comportamento Motor, tanto em Aprendizagem como em Controle Motor. Dois principais tipos de "feedback" têm sido foco de investigaçóes: a) intrínseco ou inerente, relacionado às informaçôes (proprioceptivas e exteroceptivas) advindas dos vários sistemas sensoriais; e b) extrínseco ou aumentado [conhecimento de resultado (CR) e conhecimento de performance
$(\mathrm{CP})$ ], referente às informaçóes sobre o desempenho de uma habilidade motora advindas de uma fonte externa ao indivíduo.

Sobre o primeiro tipo de "feedback", a pósgraduação da EEFE-USP mostrou que: a) os indivíduos são capazes de corrigir seus movimentos em tarefas de interceptação com base na visão periférica, embora o fluxo visual exija maior latência para promover a integração visuomotora ${ }^{31}$; b) não somente a velocidade e o nível de incerteza envolvidos no deslocamento de um objeto afetam o comportamento visual, mas, também o conhecimento acerca das características de deslocamento do objeto ${ }^{32}$; c) a ausência ou presença de informação visual não perturba o desempenho em habilidades motoras rítmicas quanto a frequência na coordenação de movimentos, o que ressalta a capacidade do sistema sensório motor de gerar sinais para sincronização a partir de um temporizador intrínseco; porém, a introdução de informação auditiva implica em transição de $\mathrm{fase}^{33}$; d) a execução de tarefas duais implica em maior demanda de atenção do que tarefas simples; contudo, tal demanda é diminuída com a prática, resultando em similar aprendizagem àquela de tarefas simples ${ }^{34}$; e) a prática afeta o período de latência para a utilização da informação visual em tarefas sincronizatórias, mas, o tempo de movimento não influencia o nível de precisão do desempenho nas referidas tarefas ${ }^{35}$; f) em tarefas complexas, a predição do desempenho parece estar mais associada às estratégias de controle utilizadas no movimento do que às restriçóes espaciais $^{36}$. 
Em relação ao segundo tipo de "feedback", a pós-graduação da EEFE-USP mostrou que: a) o "feedback" extrínseco fornecido por meio de videoteipe é eficaz na aprendizagem de habilidade no voleibol ${ }^{37}$; b) a variação do intervalo pré-CR não afeta a aprendizagem ${ }^{38}$; c) a frequência de $25 \%$ de CR é mais eficiente do que a frequência de $100 \%$, independentemente da complexidade da tarefa ${ }^{39} ; \mathrm{d}$ ) as frequências de $100 \%$ e $33 \%$ de CP possibilitam aprendizagens similares ${ }^{40}$.

Se o "feedback" diz respeito à informação sobre aquilo que já foi ou está sendo feito, a instrução remete ao futuro, ou seja, a uma informação sobre o que, como, quando e/ou o quanto fazer para se alcançar uma referência futura. Nesse âmbito, as contribuiçôes da pós-graduação da EEFE-USP foram para mostrar que a instrução geral pode fornecer informação suficiente para a aprendizagem de habilidades motoras complexas ${ }^{41}$, e que ela poderia ser sobre como os componentes da habilidade interagem de modo a caracterizá-la como um todo ${ }^{42}$. Além disso, foi mostrado que as instruçôes sobre o padrão de movimento devem ser fornecidas no início da aprendizagem, enquanto que as informaçóes sobre seu resultado no ambiente devem ser focalizadas em estágios mais avançados ${ }^{43}$. E que, fornecidas em forma de dicas, as instruçóes podem constituir-se náo somente em informaçóes sobre aspectos relevantes da habilidade motora, mas, também em conhecimentos sobre elas ${ }^{44}$. Contribuiu-se, também, para a compreensão de que somente instruçóes verbais não são capazes de modificar as expectativas do aprendiz em relação às suas estratégias de desempenho ${ }^{45}$, e que as instruçóes são mais eficazes quando a verbalização é combinada com demonstraçôes de modelos humanos em vídeo ou em boneco ${ }^{46}$.

No que concerne à prática, os achados têm contribuído para refutar, em grande parte, as proposiçóes da interferência contextual e formação de esquema motor relativas à estruturação da prática. Refutou-se a hipótese de que a variabilidade de prática implicaria no fortalecimento de um esquema motor e, consequentemente, no aumento do potencial de transferência, isto é, desempenho em uma nova $\operatorname{tarefa}^{47}$. Na mesma linha, foram obtidas evidências de que o alto nível de interferência contextual não afeta positivamente a aprendizagem de habilidades motoras mais do que os níveis moderado e baixo ${ }^{48-49}$. Também foi mostrado que o efeito da interferência contextual não é dependente da interação entre as dificuldades funcional e nominal da tarefa, isto é, da experiência do praticante e da complexidade da tarefa ${ }^{50-51}$ e, tampouco, do intervalo de aplicação do teste de retençãa ${ }^{52}$. Entretanto, foi mostrado que em situação real de ensino-aprendizagem, no caso, habilidades motoras do judô, a sua aquisiçáo é beneficiada pela combinaçáo das práticas constante seguida de variada aleatória em comparação às práticas constante, variada por blocos e variada aleatória ${ }^{53}$. Ainda nesse contexto, foi mostrado que a prática próxima das condiçôes reais de luta é mais eficaz para a aprendizagem do que a prática que prioriza partes isoladas das habilidades motoras ${ }^{54}$.

Em relação a aspectos cognitivos envolvidos na execução e aprendizagem de habilidades motoras, as teses e dissertaçôes defendidas na pós-graduação da EEFE-USP mostraram que com a prática, crianças de quatro, cinco e seis anos de idade modificam diferentemente a forma de planejamento na execução de tarefas de manipulação (apreender, transportar, orientar e inserir uma barra num orifício semicircular), porém, melhoram o desempenho de maneira similar ${ }^{55}$. E que a idade afeta a capacidade de crianças e adolescentes de estimarem intervalos de tempo de 10 e 30 segundos na execução de habilidades motoras do basquetebol ${ }^{56}$.

Contrariando a literatura, evidências foram obtidas de que não há correlação entre o nível de experiência e o desempenho cognitivo (nível de conhecimento medido, capacidade de lembrança de posicionamento e repertório de alternativas pertinentes) de jogadores de handebol ${ }^{57}$. Na mesma linha, foi mostrado que os jogadores de futsal, independentemente dos níveis de experiência e de conhecimento, baseiam-se nas informaçóes relativas às relaçóes interpessoais angulares (interaçáo de vetores ligando o portador da bola com seu companheiro de time e o marcador dele) para tomar decisôes sobre a realização da habilidade passe ${ }^{58}$. Além disso, mostrou-se que as decisões de chutar e driblar no futebol são tomadas, respectivamente, com base nas informaçóes sobre as relaçôes angulares representando a interação entre as possibilidades de o chute ser completado e interceptado, e na distância interpessoal ${ }^{59}$. E, que o levantador de voleibol decide largar ao invés de levantar com base em informações sobre a área formada entre oponentes, a distância e a velocidade de aproximação dele em relação à rede, a velocidade da bola, e a distância entre ele e os bloqueadores ${ }^{60}$.

No tocante à transferência lateral e/ou coordenação bimanual, as teses e dissertações possibilitaram avanços em relaçáo à compreensão dos desempenhos entre os membros dominante 
e não dominante, e sobre como a prática com ênfase em um pode ser transferida para o outro. Mostrou-se que: a) a transferência interlateral de aprendizagem de habilidades motoras não é afetada pelo tipo de prática (física, mental, e física e mental em conjunto), ou seja, a aprendizagem de habilidades motoras com demanda de movimentos bilateralmente coordenados é específica ao lado praticado ${ }^{61}$; b) a preferência podal e as assimetrias interlaterais de desempenho são similares entre crianças de diferentes faixas etárias com prática regular em açóes motoras relacionadas ao futebol, e a preferência podal é dependente da tarefa (estabilização e mobilização) ${ }^{62}$; c) as assimetrias interlaterais são acentuadas em frequências de movimento mais altas e o melhor desempenho com o braço dominante está relacionado ao modo de coordenação mais consistente e efetivo em realizar a trajetória de movimento desejada ${ }^{63-64}$.

Com respeito à postura, as teses e dissertaçóes da EEFE-USP mostraram que: a) a previsibilidade de perturbaçóes externas ao corpo, as demandas espaciais da habilidade e o "feedback" intrínseco influenciam o controle postural ${ }^{65-66}$; b) a realização de tarefas cognitivas e de apontamento manual afetam, negativa e positivamente, a manutenção da postura em equilíbrio; no caso negativo, isso pode ter ocorrido devido à competição de recursos para realizar as tarefas ${ }^{67}$; c) as informaçóes contextuais modulam respostas posturais reativas, e o controle postural em indivíduos idosos é beneficiado por informaçóes contextuais de forma similar a adultos jovens $s^{68}$; d) a dinâmica intrínseca do sistema, referente ao relacionamento entre informação visual e oscilação corporal, não é facilmente modificada pela informação comportamental; enquanto adultos jovens utilizam as informaçóes fornecidas de forma mais adequada para a ação solicitada, crianças apresentam dificuldade em utilizar a informação fornecida ou realizar uma ação solicitada frente à dinâmica intrínseca do sistema ${ }^{69}$.

Embora a motivação seja reconhecida como um importante fator que afeta o comportamento motor e, em específico, a aprendizagem motora, apenas dois trabalhos focalizaram-na no programa de pósgraduaçáo da EEFE-USP. Eles mostraram que os estabelecimentos de metas genéricas e específicas de curto e longo prazo afetam similarmente a aprendizagem de habilidades motoras ${ }^{70}$. Além disso, mostraram que a percepção de competência influencia a aprendizagem motora de maneira que, indivíduos com alta percepção de competência aprendem melhor do que indivíduos de baixa percepção de competência. Mas, que o inverso não ocorre, isto é, a aprendizagem motora não afeta a percepção de competência ${ }^{71}$.

O desenvolvimento motor é um dos temas que há mais tempo tem sido foco de teses e dissertaçôes na área de Comportamento Motor na pósgraduação da EEFE-USP. As suas contribuiçóes têm ocorrido em relação a três principais preocupações: a) desenvolvimento de padrôes fundamentais de movimento frente a diferentes restrições ("constraints"); b) caracterização do desenvolvimento motor em distintos ambientes; e c) relacionamento entre o desenvolvimento motor e atividade física.

Com relação à primeira preocupação, as teses e dissertações evidenciaram a importância do estágio de desenvolvimento motor na organização do padrão arremessar, considerando as mudanças no objetivo da tarefa ${ }^{72}$. Mostraram também que: a) crianças de diferentes idades realizaram ajustes similares no movimento da perna dominante na habilidade de $\operatorname{chutar}^{73}$; b) mudanças no nível de complexidade da tarefa restringem o padrão de preensão manual (palmar e digital) de crianças nos dois primeiros anos de vida ${ }^{74}$; ) a prática sistemática influencia o modo com o qual crianças de diferentes estágios de desenvolvimento na habilidade motora básica rebater controlam os graus de liberdade articulares $^{75}$; d) mesmo após os sete anos de idade, ocorrem melhoras na qualidade das habilidades motoras básicas, apesar de elas serem específicas à classe de movimento (locomoçáo e manipulação) e ao $\operatorname{sexo}^{76}$; e) grande parte dos adultos não apresenta níveis maduros de desenvolvimento motor na habilidade básica arremessar ${ }^{77}$.

Concernente à segunda preocupação, as teses e dissertaçôes mostraram que: a) crianças de 12 anos de idade, moradores de regióes urbanas do Chile, apresentam padrôes de "hop" menos avançados do que aquelas de regióes montanhosas, sendo os meninos mais avançados do que as meninas, e que tais diferenças não ocorrem em relação às crianças de seis anos de idade ${ }^{78}$; b) em ambientes livres da educação infantil, crianças de quatro e cinco anos de idade permanecem mais tempo realizando habilidades posturais seguidas de manipulação e locomoção; quando brincam, meninos e meninas diferem mais, respectivamente, em relação ao futebol e à fantasia ${ }^{79}$; c) em ambiente de brinquedoteca, as crianças realizam as três categorias de açôes, de estabilidade, de manipulação e de locomoção, os brinquedos utilizados são carrinhos, utensílios 
domésticos e bonecos (as) e tanto meninos quanto meninas permanecem na maioria do tempo sozinhos $^{80 ;}$ d) crianças das zonas rural e urbana de Manaus-AM mostram desempenhos semelhantes, tanto no escore total do teste MABC, quanto nos testes específicos das habilidades manuais, com bola e de equilíbrio deste mesmo teste ${ }^{81}$.

E, com relação à terceira preocupaçáo, quatro trabalhos foram realizados com base num estudo longitudinal misto de crescimento e desenvolvimento motor realizado em Muzambinho-MG. Eles mostraram que: a) a maioria das crianças da referida cidade apresenta desempenho em habilidades motoras abaixo do esperado considerando o teste TGMD-2, e que tais habilidades poderiam ser preditoras dos níveis de atividade física ao longo do tempo ${ }^{82}$; b) não há associação entre os resultados dos testes TGMD-2 e o KTK na avaliação motora, independentemente de idade e sexo das crianças ${ }^{83}$; c) o desempenho em tarefas motoras típicas da infância está associado às capacidades perceptivo-motoras, mas o nível de maturação do sistema nervoso central pode ser um aspecto que interfere nesta associação ${ }^{84}$; d) não há associação entre coordenação motora e atividade física, pois, enquanto a primeira melhorou ao longo do tempo, a segunda se manteve ${ }^{85}$.

No tocante à categoria deficiência, as teses e dissertaçôes da pós-graduação da EEFE-USP mostraram que: a) o diagnóstico parcial de crianças com transtorno do desenvolvimento da coordenação, por professores de sala e de educação física, por meio da lista de checagem do teste MABC do movimento, é possível quando considerados complementarmente ${ }^{86}$; b) as referidas crianças apresentam tempo para o planejamento de tarefas com exigência de precisão similarmente às crianças com desenvolvimento típico, embora o façam com maior frequência $\left.{ }^{87} ; c\right)$ além disso, crianças com graus leve e severo do referido transtorno apresentam dificuldades na formação de programas de ação de habilidades gráficas, e o programa formado possibilita similar adaptaçáo de ambos à ausência de "feedback" visual e à mudança na forma de escrita ${ }^{88}$; d) quanto a organização temporal, crianças com o citado transtorno diferem de crianças com desenvolvimento típico apenas em relação ao "timing" sincronizatório ${ }^{89}$; e) os componentes de aptidão física relacionados à saúde não são fatores que diferenciam crianças com níveis severo e moderado de transtorno do desenvolvimento da coordenaçáa ${ }^{90}$; f) crianças com características de transtorno do desenvolvimento da coordenaçáo submetidas a intervençóes com atividades aquáticas e terrestres alcançam desempenhos similares às crianças com desempenhos típicos ${ }^{91}$; g) crianças com transtorno do déficit de atenção e hiperatividade podem apresentar déficits motores independentemente de haver co-ocorrência com o transtorno do desenvolvimento da coordenaçãa ${ }^{92}$.

Avançou-se, também, no conhecimento em relaçáo a indivíduos que sofreram acidente vascular cerebral (I-AVC). As teses e dissertaçôes da pósgraduação da EEFE-USP mostraram que: a) I-AVC com lesão no hemisfério esquerdo apresentam maiores dificuldades na aprendizagem de habilidades motoras com demanda de planejamento, em comparação com indivíduos cuja lesão é no lado direito ${ }^{93}$; b) independentemente do hemisfério lesado, os referidos indivíduos aprendem igualmente uma tarefa de controle postural, porém, em níveis inferiores a indivíduos neurologicamente saudáveis; mas, a complexidade da tarefa é um fator de dificuldade para os indivíduos com a lesão no hemisfério direito ${ }^{94-95}$; c) a prática mental influencia positivamente e igualmente a aprendizagem de uma tarefa com exigências de velocidade e precisão por indivíduos com lesão no hemisfério direito e esquerdo ${ }^{96}$; d) apesar do comprometimento no equilíbrio, I-AVE mostram vantagem do hemisfério cerebral direito no controle da postura quieta e em perturbação sensorial ${ }^{97}$; e) a manipulação das informaçôes sensoriais visual e tátil ao longo da prática favorece a estabilização, a recuperação e a melhora do equilíbrio corporal de I-AVC ${ }^{98} \mathrm{f}$ ) o fornecimento de $\mathrm{CP}$ promove melhor aprendizagem motora do que o $\mathrm{CR}^{99}$.

Além disso, as teses e dissertações mostraram que: a) crianças com síndrome de Down leve e moderada se beneficiam igualmente da variabilidade de prática na aprendizagem de uma tarefa de arremessar ao alvo $^{100}$; b) enquanto crianças de grau moderado não foram capazes de combinar habilidades motoras básicas, aquelas com grau de comprometimento leve apresentaram padróes similares aos das crianças normais. E, por fim, foi mostrado que indivíduos surdos aprendem melhor quando recebem dicas visuais em comparação a dicas cinestésicas e dicas visuais e cinestésicas em conjunto ${ }^{102}$.

Referente aos idosos, as teses mostraram que: a) idosos tenistas são suscetíveis aos efeitos da especificidade da tarefa, visto apresentarem desempenho numa tarefa de coincidência temporal similar a adultos jovens, e superior a idosos não praticantes ${ }^{103}$; b) idosos apresentam características 
cinemáticas similares aos adultos em relação ao padrão de marcha regular, sendo que quando o comprimento do passo é alterado, ambos modificam igualmente a velocidade de deslocamento, a amplitude de movimentação do membro superior e a relação de fase entre membro superior e inferior ${ }^{104}$; c) não há correlação entre a estrutura do pé (mais precisamente o arco longitudinal medial) e o equilíbrio em idosos praticantes e não praticantes de futsal as variáveis pé e equilíbrio tanto em diferentes tipos de índice de massa corporal quanto em idosos praticantes e não praticantes de futsal ${ }^{105}$; d) idosos sadios apresentam diferentes padrões de coordenação ombro-quadril em função da restrição da tarefa, enquanto idosos com doença de Parkinson apresentam um padrão relativamente invariável, com a capacidade de adaptação de respostas posturais comprometida ${ }^{106}$; e) o treinamento de força não afeta o equilíbrio de idosos em situações estáticas uni e bipodal ${ }^{107}$.

No que se refere à aprendizagem motora de idosos, as teses e dissertaçôes da pós-graduação da EEFE-USP mostraram que: a) a aprendizagem do arremesso do lance livre do basquetebol por idosos é mais beneficiada quando a prática envolve 66\% de frequência de $\mathrm{CP}^{108}$; b) diferentemente do $\mathrm{CP}$, o CR não exerce suficientemente suas funçóes ao ponto de promover a aprendizagem de idosos, independentemente da frequência $(100 \%$, 66\% e $33 \%)^{109}$; c) idosos se beneficiam igualmente das instruçóes relativas aos focos interno e externo de atenção ${ }^{110}$, mas não o fazem em relação ao efeito da interferência contextual ${ }^{111}$; d) a prática sistemática de exercícios e esportes é eficaz em promover melhorias em componentes de aptidão física relacionados à saúde e à habilidade, mas não é capaz de causar mudanças nas estruturas cerebrais ${ }^{112}$.

Sobre a categoria de autocontrole, as teses e dissertaçóes mostraram que: a) contrariamente aos resultados com adultos, crianças com controle externo obtêm melhores resultados de aprendizagem, e não são tão eficientes quanto aos adultos em discriminar entre boas e más tentativas para a solicitação de $\mathrm{CR}^{113}$; b) o autocontrole de $\mathrm{CP}$ não afeta a aprendizagem de idosos; o CP prescritivo promove mudanças no padrão de movimento, bem como na diminuição dos erros; além disso, a frequência de CP elevada ou 55\% parece ser um fator que incrementa o desempenho de idosos ${ }^{114}$; c) a demonstração autocontrolada produz melhor aprendizagem do que a condiçáo externamente controlada, sendo o tempo para a tomada de decisão e a quantidade de demonstração, fatores determinantes dessa superioridade ${ }^{115}$; d) para iniciantes, ter controle sobre a auto-observação (observar o melhor desempenho ou o desempenho global) possibilita melhor aprendizagem; para aprendizes em estágio intermediário, o controle sobre a auto-observação só é eficaz com o aumento da prática; mudar a escolha não implicou em melhor aprendizagem; e, a auto-observação melhora a crença de auto eficácia independente da escolha ${ }^{116}$; e) a informaçáo sobre meta de aprendizagem na aprendizagem autocontrolada influencia a forma com que os aprendizes organizam sua prática ${ }^{117}$; f) a autofala não influencia a aprendizagem do "forehand" do tênis de campo, independentemente da quantidade e do componente verbalizado ${ }^{118}$.

Conforme descrito anteriormente, habilidades motoras aquáticas foram foco de algumas teses e dissertaçóes. Elas mostraram que: a) a prática sistemática afeta a locomoção aquática no primeiro ano de vida no que concerne aos componentes cabeça, braços, pernas e tronco do mergulho ventral autônomo ${ }^{119}$; b) o estágio de desenvolvimento relativo à locomoção aquática é modificado em direção a padrôes rudimentares de locomoção como consequência de restriçóes ambientais; quando as restrições envolvem mudanças relativas à direção e à velocidade da locomoção, os indivíduos mais avançados adaptam-se via alteraçóes na sequência das açóes e em seus parâmetros; e, quando apenas a velocidade é modificada, a adaptação envolve apenas alteração nos parâmetros da ação ${ }^{120}$; c) é viável a construção de um aparato de suporte à locomoção aquática de bebês e de crianças de três a 24 meses de idade ${ }^{121}$.

As teses e dissertaçóes mostraram também que: a) crianças habilidosas no nado crawl mantêm a organização temporal relativa da braçada em diferentes velocidades do nado, mas alteram a referida organização em termos de parâmetros ${ }^{122}$; b) o mesmo cabe para atletas de nível nacional em comparação com aqueles de nível estadual ${ }^{123}$; c) diferentemente, em ambientes instáveis como o mar, nadadores habilidosos efetuam ajustes em ambas as dimensóes da organização temporal da braçada ${ }^{124}$; d) o padrão preferido de respiração não necessariamente corresponde ao desempenho mais eficiente e tampouco altera a estrutura temporal relativa da braçada ${ }^{125}$, mas e) ele afeta o desempenho quando considerado em associação com a coordenação da braçada ${ }^{126}$.

Por fim, a última categoria foi formada a partir de uma concepção particular de aprendizagem 
motora: o modelo de processo adaptativo. As teses e dissertaçóes mostraram que: a) a aquisição de habilidades motoras caracteriza-se como um processo adaptativo envolvendo sucessivos ciclos de estabilização-adaptação, em que a redundância inicial do sistema e a magnitude da perturbação têm efeitos no desempenho ${ }^{127}$; b) independentemente do nível de estabilização funcional, a perturbação causada por mudanças perceptivo-motoras na tarefa é superior àquela somente motora e perceptiva, e que as alteraçóes motoras são mais perturbadoras do que as perceptivas ${ }^{128}$; c) um programa de ação organizado hierarquicamente em macro e microestruturas pode emergir como hipótese explicativa sobre como novas habilidades poderiam ser formadas a partir da reorganização das anteriores (processo adaptativo) ${ }^{129}$; d) a variabilidade nessas estruturas teria papeis diferenciados conforme o estado de organização do sistema: garantir a configuração e a consistência nas açóes (macro) e possibilitar-lhe flexibilidade (micro) ${ }^{130}$; e) mesatenistas adaptam suas rebatidas via reorganização (alteração da macroestrutura) quando a dificuldade da rebatida é alta ou, por meio da flexibilidade da microestrutura, quando o nível de dificuldade é baixo ${ }^{131}$.

As teses e dissertaçôes mostraram também que: a) a modificação estrutural e uma ou mais unidades básicas da açáo (componentes) gera superior desempenho em comparação com a modularização das citadas unidades ${ }^{132}$; b) a modularização é facilitada quando o desempenho estabiliza em canais superiores de consistência ${ }^{133}$; c) as diferenças individuais são responsáveis por uma parcela da heterogeneidade do desempenho observado no processo adaptativo ${ }^{134}$.

\section{Considerações finais}

A pós-graduação é um sistema de ensino dinâmico e complexo. A natureza dinâmica implica em os programas (cursos) necessitarem estar sintonizados com as mudanças que ocorrem na sociedade a fim de se adaptarem e lograrem suas finalidades. O olhar para trás para acessar o que foi feito, bem como para frente, para vislumbrar o que necessita ainda ser realizado, remetem a aspectos fundamentais para a referida adaptação. Por sua vez, a natureza complexa implica na necessidade de o sistema ser analisado por diferentes olhares, isto é, por diferentes abordagens e níveis análise.
Por fim, as teses e dissertações da pós-graduação da EEFE-USP mostram que: a) um alto grau de liberdade na escolha durante a estabilização de uma habilidade motora prejudica o processo de adaptação a uma modificação perceptivo-efetora da tarefa e o não fornecimento de liberdade de escolha prejudica a adaptação a uma modificação efetora da tarefa ${ }^{135-136}$; b) a liberdade na escolha das respostas com o esclarecimento da meta de aprendizagem não é suficiente para beneficiar o processo adaptativo ${ }^{137}$; c) a estrutura de prática constante com liberdade na escolha de alguns componentes para formar a sequência proporciona melhor adaptação ${ }^{138}$; d) há efeitos benéficos das práticas constante-variada por blocos $^{139}$ e aleatória ${ }^{140}$ no processo adaptativo de aprendizagem motora; e) há complementaridade de especificidade/generalidade da tarefa nos efeitos destas estruturas de prática ${ }^{30} ;$ f) há uma quantidade mínima, ótima, de variabilidade na prática aleatória, após a prática constante, para a ocorrência do processo adaptativo ${ }^{141} ; g$ ) os efeitos das práticas constante, aleatória, constante-aleatória e aleatória-constante no processo adaptativo de aprendizagem motora não são afetados pelo nível de validade ecológica da situação de aprendizagem ${ }^{142}$; h) o processo adaptativo na aprendizagem de crianças, adultos e idosos de uma tarefa de "timing" coincidente é mais beneficiado pela prática constante-aleatória ${ }^{143}$; i) o processo adaptativo independe ${ }^{144}$, mas depende da complexidade da $\operatorname{taref}^{145} ; \mathrm{j}$ ) a associação da estrutura de prática e da frequência de CP náo possibilita o processo adaptativo na aprendizagem do saque por cima do voleibol $^{146}$.
$\mathrm{O}$ presente texto delimitou-se às contribuiçóes da pós-graduaçáo da EEFE-USP, em seus 40 anos existência, para o avanço do conhecimento na subárea de Comportamento Motor.

Ao longo de seus 40 anos, a pós-graduação da EEFE-USP formou recursos humanos com o domínio do conhecimento e do método científico em Controle, Aprendizagem e Desenvolvimento Motor, que possibilitaram investigar questôes relativas à novidade, variabilidade motora, consistência motora e equivalência motora. Mais especificamente, a pósgraduação da EEFE-USP tem contribuído para o 
avanço do conhecimento sobre os seguintes temas: "feedback", prática, idoso, instrução, deficiência, desenvolvimento, cognição, postura, transferência lateral, habilidades aquáticas, processo adaptativo, autocontrole, atenção/visão e motivação.

Embora o objetivo da pós-graduação da EEFEUSP seja a formação de docentes, pesquisadores e profissionais, é na docência em instituiçôes de ensino superior que a maioria dos egressos tem atuado ${ }^{6}$. Isto significa a efetiva contribuição do programa no que se refere à nucleação, formando recursos humanos qualificados para instituiçóes de todas as regióes do Brasil, além de algumas universidades estrangeiras. Vale destacar, finalmente, a importância da pós-graduação da EEFE-USP no desenvolvimento da subárea de Comportamento Motor, visto que está no Laboratório de Comportamento Motor da EEFE-USP a gênese da Sociedade Brasileira de Comportamento Motor.

\section{Referências}

1. Goldemberg J. USP 80 anos. São Paulo: Edusp; 2015.

2. Motoyama S, organizador. USP 70 anos: imagens de uma história vivida. São Paulo: Edusp; 2006.

3. Henry FM. Physical education: an academic discipline. JOHPER. 1964;35:32-8,69.

4. Henry FM. The academic discipline of physical education. Quest. 1978;29:13-29.

5. Tani G. Cinesiologia, educação física e esporte: ordem emanente do caos na estrutura acadêmica. Motus Corporis. 1996;3:9-49.

6. Tani G. Pós-graduação em educação física: crescimento e correção da rota. In: Moreira WW, Nista-Piccolo VL, organizadores. Educação física e esporte no século XXI. Campinas: Papirus Editora; 2016. p.153-71.

7. Corrêa UC. Pesquisa em comportamento motor: a intervenção profissional em perspectiva. São Paulo: EFP-EEFUSP; 2008.

8. Pellegrini AM, Hiraga CY, Alleoni BN., Silva LH. Comportamento motor no Brasil: um olhar para o passado, pensando no futuro. Braz J Motor Behav. 2006;1:32-40.

9. Tani G. Comportamento motor e sua relação com a educação bísica. Braz J Motor Behav. 2006;1:20-31.

10. Edwards WH. Motor learning and control: from theory to practice. Belmont: Wadsworth; 2010.

11. Kelso JAS. Human motor behavior: an introduction. Hillsdale: Lawrence Erlbaum; 1982.

12. Tani G, Manoel EJ. Esporte, educação física e educação física escolar. In: Gaya AC, Marques AT, Tani G, organizadores. Esporte para crianças e jovens: razóes e finalidades. Porto Alegre: Universidade Federal do Rio Grande do Sul; 2004. p.45-60.

13. Tani G. Comportamento motor: aprendizagem e desenvolvimento. Rio de Janeiro: Guanabara Koogan; 2005.

14. Tani G. Comportamento motor: conceitos, estudos e aplicaçôes. Rio de Janeiro: Guanabara oogan;. 2016.

15. Adams JA. Historical review and appraisal of research on the learning, retention, and transfer of human motor skills. Psychol Bull. 1987;101:41-74.

16. Clark JE, Oliveira MA. Motor behavior as a scientific field: a view from start of the 21 st century. Braz J Motor Behav. 2006;1:1-19.

17. Connolly KJ. Desenvolvimento motor: passado, presente e futuro. Rev. Paul Educ Fís. 2000;Supl.3:6-15.

18. Schmidt RA. Motor control and learning: a behavioral emphasis. 2nd ed. Champaign: Human Kinetics; 1988.

19. Thomas JR. Motor behavior: from telegraph keys and twins to linear slides and stepping. Quest. 2006;58:112-27.

20. Cratty BJ. Movement behavior and motor learning. Philadelphia: Lea \& Febiger; 1964.

21. Graint R. The basis of motor control. New York: Academic Press; 1970.

22. Connolly KJ. Mechanisms of motor skill development. London: Academic Press; 1970.

23. Adams JA. A closed-loop theory of motor learning. J Motor Behav. 1971;3:111-50.

24. Schmidt RA. A schema theory of discrete motor skill learning. Psychol Rev. 1975;82:225-60.

25. Sherrington CS. The integrative action of the nervous system. New Haven: Yale University Press; 1906.

26. Bryan WL, Harter N. Studies in the physiology and psychology of the telegraphic language. Psychol Rev. 1897;4:27-53.

27. Tiedemann D. Observations on the mental development of a child. New York: Appleton-Century-Crofts; 1787. (Historical Readings in Developmental Psychology).

28. Fischman MG. Motor learning and control foundations of kinesiology: defining the academic core. Quest. 2007;59:67-76.

29. Krebs RJ. A teia do conhecimento produzido em comportamento motor e ciência do desenvolvimento humano. In: Corrêa UC. Pesquisa em comportamento motor: a intervenção profissional em perspectiva. São Paulo: EFP-EEFEUSP; 2008. p.26-40. 
30. Barros JAC. Estrutura de prática e processo adaptativo em aprendizagem motora: efeitos da especificidade da tarefa [dissertação]. São Paulo: Universidade de São Paulo, Escola de Educação Física e Esporte; 2006.

31. Caminha LQ. Interceptação de alvos móveis: uso de informação visual fornecida por diferentes sistemas de detecção de movimento [dissertação]. São Paulo: Universidade de São Paulo, Escola de Educação Física e Esporte; 2008.

32. Siqueira NS. Perseguição visual em ações interceptativas em situaçóes de incerteza sobre a direçáo de deslocamento do alvo [dissertação]. São Paulo: Universidade de São Paulo, Escola de Educação Física e Esporte; 2012.

33. Mortari KSM. Integração sensório motora: um estudo sobre coordenação bimanual [dissertação]. São Paulo: Universidade de São Paulo, Escola de Educação Física e Esporte; 2001.

34. Claudio APK. Aprendizagem em tarefas duais: variação de desempenho e demanda atencional [dissertação]. São Paulo: Universidade de São Paulo, Escola de Educação Física e Esporte; 2011.

35. Teixeira LA. Integração visomotora no controle de tarefas sincronizatórias [tese]. São Paulo: Universidade de São Paulo, Escola de Educação Física e Esporte; 1995.

36. Okazaki VHA. Controle de movimentos rápidos e precisos direcionados a alvos espaciais [tese]. São Paulo: Universidade de São Paulo, Escola de Educação Física e Esporte; 2009.

37. Jesus JF. O efeito do feedback extrínseco fornecido através do videotape na aprendizagem de uma habilidade motora do voleibol [dissertação]. São Paulo: Universidade de São Paulo, Escola de Educação Física; 1986.

38. Proença JE. Efeitos da variação temporal do conhecimento de resultado na aprendizagem de uma habilidade motora discreta [dissertação]. São Paulo: Universidade de São Paulo, Escola de Educação Física; 1989.

39. Oliveira DL. Frequência relativa de conhecimento de resultados e complexidade da tarefa na aprendizagem de uma habilidade motora [dissertação]. São Paulo: Universidade de São Paulo, Escola de Educação Física e Esporte; 2002.

40. Martel VSA. Efeitos da freqüência de conhecimento de performance na aprendizagem de habilidades motoras [dissertação]. São Paulo: Universidade de São Paulo, Escola de Educação Física e Esporte; 2005.

41. Denardi RA. Efeitos do foco de instruçáo relacionado ao componente da tarefa durante a aprendizagem da pirouette do balé clássico [dissertação]. São Paulo: Universidade de São Paulo, Escola de Educaçáo Física e Esporte; 2011.

42. Marques MTSP. Dicas verbais na aprendizagem de uma habilidade motora: foco no componente e na interaçáo entre os componentes [dissertação]. São Paulo: Universidade de São Paulo, Escola de Educação Física e Esporte; 2012.

43. Oliveira TAC. Efeitos dos focos interno e externo de atençáo na aprendizagem de habilidades motoras [dissertação]. São Paulo: Universidade de São Paulo, Escola de Educação Física e Esporte; 2010.

44. Silveira SR. Aquisição de habilidades motoras na educação física escolar: um estudo das dicas de aprendizagem como conteúdo de ensino [tese]. São Paulo: Universidade de São Paulo, Escola de Educação Física e Esporte; 2010.

45. Azevedo Neto RM. Efeito da expectativa na reorganização das dimensôes espacial e temporal em açóes interceptativas [dissertação]. São Paulo: Universidade de São Paulo, Escola de Educação Física e Esporte; 2012.

46. Fugita M. Efeitos do modelo na aprendizagem do nado sincronizado [tese]. São Paulo: Universidade de São Paulo, Escola de Educação Física e Esporte; 2010.

47. Passos SCE. Efeitos da variabilidade de prática na aprendizagem de uma habilidade motora [dissertação]. São Paulo: Universidade de São Paulo, Escola de Educação Física; 1989.

48. Meira Junior CM. O efeito da interferência contextual na aquisição da habilidade saque do voleibol em crianças: temporário, duradouro ou inexistente [dissertação]. São Paulo: Universidade de São Paulo, Escola de Educação Física e Esporte; 1999.

49. Ugrinowitsch H. Interferência contextual: manipulação de programas e parâmetros na aquisição da habilidade saque do voleibol [dissertação]. São Paulo: Universidade de São Paulo, Escola de Educação Física e Esporte; 1997.

50. Babo AGF. Interferência contextual na aprendizagem de habilidades gráficas em crianças: adaptação ipsi e contralateral [dissertação]. São Paulo: Universidade de São Paulo, Escola de Educação Física e Esporte; 2008.

51. Souza TO. O efeito da interferência contextual na aquisição de habilidades motoras em função das dificuldades funcional e nominal da tarefa [dissertação]. São Paulo: Universidade de São Paulo, Escola de Educação Física e Esporte; 2012.

52. Santos JJ. Efeitos da interferência contextual na aprendizagem de habilidades motoras em função de diferentes testes de retenção [dissertação]. São Paulo: Universidade de São Paulo, Escola de Educação Física e Esporte; 2012.

53. Gomes FRF. Estrutura de prática na aprendizagem do golpe de Judô o soto gari: foco no kuzuzhi [tese]. São Paulo: Universidade de São Paulo, Escola de Educaçâo Física e Esporte; 2016.

54. Gomes FRF. Aprendizagem do kuzushi (desequilíbrio) nos golpes de judô o soto gari e tai otoshi [dissertação]. São Paulo: Universidade de São Paulo, Escola de Educação Física e Esporte; 2007.

55. Moreira CRP. Efeito da prática no planejamento de uma habilidade de manipulação em crianças na primeira infância [dissertação]. São Paulo: Universidade de São Paulo, Escola de Educação Física e Esporte; 2002.

106 • Rev Bras Educ Fís Esporte, (São Paulo). 2017 Ago; 31(N esp):97-110. 
56. Schonardie Filho L. A aprendizagem da estimativa de duração do tempo em função da idade e sexo [dissertação]. São Paulo: Universidade de São Paulo, Escola de Educação Física; 1989.

57. Dantas LEPBT. Conhecimento no desempenho de habilidades motoras [dissertaçáo]. São Paulo: Universidade de São Paulo, Escola de Educação Física e Esporte; 2000.

58. Silva SL. O efeito da experiência, do conhecimento e da habilidade na tomada de decisão do passe do futsal a partir das relaçôes interpessoais angulares [dissertação]. São Paulo: Universidade de São Paulo, Escola de Educação Física e Esporte; 2014.

59. Romero Clavijo FA. Relações espaço-temporais como restrições de tomadas de decisóes na grande área no futebol [dissertação]. São Paulo: Universidade de São Paulo, Escola de Educação Física e Esporte; 2016.

60. Denardi RA. A decisão da largada do levantador do voleibol na perspectiva de dinâmica ecológica [tese]. São Paulo: Universidade de São Paulo, Escola de Educação Física e Esporte; 2015.

61. Castellano RMC. Transferência bilateral de aprendizagem em habilidades motoras globais: efeito da prática mental [dissertação]. São Paulo: Universidade de São Paulo, Escola de Educação Física e Esporte; 2000.

62. Teixeira MCT. Preferência podal e assimetrias interlaterais de desempenho na tarefa de chutar em crianças [dissertação]. São Paulo: Universidade de São Paulo, Escola de Educação Física e Esporte; 2007.

63. Pereira, C.F. Assimetrias interlaterais na coordenação interarticular de movimentos manuais circulares cíclicos [dissertação]. São Paulo: Universidade de São Paulo, Escola de Educação Física e Esporte; 2011.

64. Monfredini CFP. Assimetrias interlaterais na coordenação interarticular de movimentos manuais circulares cíclicos [dissertação]. São Paulo: Universidade de São Paulo, Escola de Educação Física e Esporte; 2010.

65. Pardini, A.C.L. Efeito de diferentes tipos de tarefa suprapostural em respostas posturais reativas a uma perturbação mecânica [tese]. São Paulo: Universidade de São Paulo, Escola de Educação Física e Esporte; 2009.

66. Lima ES. Integração do controle postural e de açóes manuais em função da previsibilidade de perturbação e da demanda de precisão espacial [dissertação]. São Paulo: Universidade de São Paulo, Escola de Educação Física e Esporte; 2008.

67. Bourlinova C. Efeito de diferentes tipos de tarefa suprapostural em respostas posturais reativas a uma perturbação mecânica [dissertação]. São Paulo: Universidade de São Paulo, Escola de Educação Física e Esporte; 2015.

68. Silva MB. Organização central na geração de ajustes posturais reativos em idosos [tese]. São Paulo: Universidade de São Paulo, Escola de Educação Física e Esporte; 2013.

69. Perotti Junior A. Efeitos da informação verbal no acoplamento entre a informação visual e oscilação corporal [tese]. São Paulo: Universidade de São Paulo, Escola de Educação Física e Esporte; 2006.

70. Souza Júnior OP. Efeitos do estabelecimento de metas na aprendizagem de habilidades motoras [dissertação]. São Paulo: Universidade de São Paulo, Escola de Educação Física e Esporte; 2005.

71. Carvalhais CKA. Percepção de competência e aprendizagem motora [tese]. São Paulo: Universidade de São Paulo, Escola de Educação Física e Esporte; 2015.

72. Oliveira JA. Estado de desenvolvimento no padrão fundamental de movimento arremessar frente a variações numa restrição da tarefa [dissertação]. São Paulo: Universidade de São Paulo, Escola de Educação Física e Esporte; 1997.

73. Oliveira RB. Análise da perna dominante do chutar de crianças: condiçóes de bola parada e em movimento [dissertação]. São Paulo (SP): Universidade de São Paulo, Escola de Educação Física e Esporte; 2011.

74. Marques I. Efeito de restriçóes da tarefa na habilidade manipulativa de crianças nos dois primeiros anos de vida [tese]. São Paulo (SP): Universidade de São Paulo, Escola de Educação Física e Esporte; 2003.

75. Oliveira JA. Aquisição de uma habilidade motora básica: a prática sistemática em foco [tese]. São Paulo: Universidade de São Paulo, Escola de Educação Física e Esporte; 2006.

76. Santos FG. Desenvolvimento dos padróes fundamentais de movimento dos 7 aos 9.5 anos de idade: um estudo centrado nas trajetórias individuais [dissertação]. São Paulo: Universidade de São Paulo, Escola de Educação Física e Esporte; 2014.

77. Sanches AB. Estágios de desenvolvimento motor em estudantes universitários na habilidade básica arremessar [dissertação]. São Paulo: Universidade de São Paulo, Escola de Educação Física; 1989.

78. Alarcón Jiménez TI. Aquisição do "HOP” em função da idade, sexo e região geográfica [dissertação]. São Paulo: Universidade de São Paulo, Escola de Educação Física; 1989.

79. Nicoletti G. Inventário das açóes motoras de crianças pré-escolares no playground [dissertação]. Sáo Paulo: Universidade de São Paulo, Escola de Educaçáo Física e Esporte; 2008.

80. Rezende DO. O brincar livre de crianças na brinquedoteca: análise da frequência de açóes motoras, tipos de brinquedos, brincadeiras e interaçôes sociais [dissertação]. São Paulo: Universidade de São Paulo, Escola de Educação Física e Esporte; 2012. 
81. Souza CJF. O teste ABC em crianças de ambientes diferentes [dissertação]. São Paulo: Universidade de São Paulo, Escola de Educação Física e Esporte; 2004.

82. Santos M. Desempenho de habilidades motoras na infância e predição dos níveis de atividade física ao longo do tempo [dissertação]. São Paulo: Universidade de São Paulo, Escola de Educação Física e Esporte; 2013.

83. Augusto FBV. Desempenho e diagnóstico motor: um estudo correlacional entre KTK e TGMD-2 [dissertação]. São Paulo: Universidade de São Paulo, Escola de Educaçáo Física e Esporte; 2015.

84. Florêncio RB. Associaçáo entre as capacidades perceptivo-motoras e o desempenho de tarefas motoras em sujeitos de 7 e 13 anos de idade [dissertação]. São Paulo: Universidade de São Paulo, Escola de Educação Física e Esporte; 2015.

85. Souza CJF. A relação entre coordenação motora e atividade física em crianças dos sete aos 10 anos de idade: um estudo longitudinal [tese]. São Paulo: Universidade de São Paulo, Escola de Educação Física e Esporte; 2011.

86. Ferreira LF. Identificação de crianças com desordens motoras: a lista de checagem do MABC em foco [dissertação]. São Paulo (SP): Universidade de São Paulo, Escola de Educação Física e Esporte; 2004.

87. Medina-Papst J. Desempenho de adultos, crianças com desenvolvimento típico e crianças com transtorno do desenvolvimento da coordenação em uma tarefa de planejamento da ação [tese]. São Paulo: Universidade de São Paulo, Escola de Educação Física e Esporte; 2015.

88. Gimenez R. Aquisição de açóes motoras em crianças com dificuldades motoras [tese]. São Paulo: Universidade de São Paulo, Escola de Educação Física e Esporte; 2006.

89. Dantas LEPBT. Perfil de crianças com transtorno do desenvolvimento da coordenação em tarefas de timing [tese]. São Paulo: Universidade de São Paulo, Escola de Educação Física e Esporte; 2006.

90. Nascimento RO. Análise da aptidáo física relacionada à saúde de crianças com transtorno do desenvolvimento da coordenação [dissertação]. São Paulo: Universidade de São Paulo, Escola de Educação Física e Esporte; 2013.

91. Ferreira TRS. Efeito da autofala na aprendizagem motora [dissertação]. São Paulo: Universidade de São Paulo, Escola de Educação Física e Esporte; 2013.

92. Goulardins JB. Perfil psicomotor de crianças com transtorno de déficit de atenção/hiperatividade do tipo combinado [tese]. São Paulo: Universidade de São Paulo, Escola de Educação Física e Esporte; 2016.

93. Torriani-Pasin C. Aprendizagem de uma habilidade motora com demanda de planejamento em pacientes pós-acidente vascular encefálico em função do lado da lesão [tese]. São Paulo: Universidade de São Paulo, Escola de Educação Física e Esporte; 2010.

94. Bonuzzi GMG. Aprendizagem de uma tarefa de controle postural de indivíduos pós-acidente vascular encefálico: efeitos da especialização hemisférica [dissertação]. São Paulo: Universidade de São Paulo, Escola de Educação Física e Esporte; 2014.

95. Palma GCS. Aprendizagem de uma habilidade motora com diferentes níveis de complexidade em sujeitos pós acidente vascular cerebral [dissertação]. São Paulo: Universidade de São Paulo, Escola de Educação Física e Esporte; 2016.

96. Sequeira ASC. Efeito da prática mental e prática física na aprendizagem motora em indivíduos com paralisia cerebral: comparação entre lesões nos hemisférios cerebrais direito e esquerdo [dissertação]. São Paulo: Universidade de São Paulo, Escola de Educação Física e Esporte; 2015.

97. Fernandes CA. Influência do hemisfério cerebral lesado e de déficits sensoriais sobre o equilíbrio corporal pós-acidente vascular encefálico [dissertação]. São Paulo: Universidade de São Paulo, Escola de Educação Física e Esporte; 2014.

98. Conterato ARM. Recuperaçáo de equilíbrio corporal após acidente vascular encefálico: ganhos imediatos de estabilidade por toque suave e de longo prazo por treinamento com restrição sensorial [tese]. São Paulo: Universidade de São Paulo, Escola de Educação Física e Esporte; 2015.

99. Soares MAA. Aprendizagem de uma habilidade motora com diferentes níveis de complexidade em sujeitos pós acidente vascular cerebral [dissertação]. São Paulo: Universidade de São Paulo, Escola de Educação Física e Esporte; 2017.

100. Pedrinelli VJ. Formação de esquema motor em crianças portadoras de síndrome de down [dissertaçáo]. São Paulo: Universidade de São Paulo, Escola de Educação Física;1989.

101. Gimenez R. Combinação de padróes fundamentais de movimento em indivíduos normais e portadores de síndrome de down [dissertação]. São Paulo: Universidade de São Paulo, Escola de Educação Física e Esporte; 2001.

102. Pasetto SC. Efeitos do fornecimento de dicas na aprendizagem de habilidades motoras do indivíduo com surdez [tese]. São Paulo: Universidade de São Paulo, Escola de Educação Física e Esporte; 2017.

103. Silva JB. Controle motor no idoso: impacto de atividades motoras prévias [dissertação]. São Paulo: Universidade de São Paulo, Escola de Educação Física e Esporte; 2004.

104. Mendes EF. Comparação de parâmetros cinemáticos da marcha entre indivíduos jovens e idosos em tarefas de alteração do comprimento preferido do passo [dissertação]. São Paulo: Universidade de São Paulo, Escola de Educação Física e Esporte; 2005.

108 • Rev Bras Educ Fís Esporte, (São Paulo). 2017 Ago; 31(N esp):97-110. 
105. Oliveira MF. Relação entre a estrutura do pé e o equilíbrio em idosos [dissertação]. São Paulo: Universidade de São Paulo, Escola de Educação Física e Esporte; 2006.

106. Pardini ACL. Adaptabilidade de respostas posturais reativas em função de restriçáo imposta por tarefa voluntária: efeito do envelhecimento e da doença de Parkinson [tese]. São Paulo: Universidade de São Paulo, Escola de Educação Física e Esporte; 2013.

107. Sousa PN. Efeito do treinamento de força na estabilidade postural de mulheres idosas [dissertaçáo]. São Paulo: Universidade de São Paulo, Escola de Educação Física e Esporte; 2006.

108. Nunes MES. Efeito da frequência de conhecimento de performance na aprendizagem motora em idosos [dissertação]. São Paulo: Universidade de São Paulo, Escola de Educação Física e Esporte; 2010.

109. Gehring PR. Freqüência de conhecimento de resultados na aquisição de uma habilidade motora em idosos [dissertação]. São Paulo: Universidade de São Paulo, Escola de Educação Física e Esporte; 2008.

110. Franzoni MM. O efeito do foco de atenção na aprendizagem motora de idosos [dissertação]. São Paulo: Universidade de São Paulo, Escola de Educação Física e Esporte; 2011.

111. Souza MGTX. A aprendizagem motora de idosos na perspectiva do efeito da interferência contextual [dissertação]. São Paulo: Universidade de São Paulo, Escola de Educação Física e Esporte; 2014.

112. Fonseca MCO. Efeitos de dois programas de exercícios físicos nas capacidades funcionais e estruturas cerebrais de idosas [dissertação]. São Paulo: Universidade de São Paulo, Escola de Educaçáo Física e Esporte; 2010.

113. Chiviacowski CS. Efeitos da freqüência do conhecimento de resultados na aprendizagem de uma habilidade motora em crianças [dissertação]. São Paulo: Universidade de São Paulo, Escola de Educação Física e Esporte; 1993.

114. Nunes MES. Efeito do conhecimento de performance autocontrolado na aquisição de uma habilidade motora em idosos [tese]. São Paulo: Universidade de São Paulo, Escola de Educação Física e Esporte; 2015.

115. Bruzi AT. Efeitos da demonstração autocontrolada na aprendizagem motora [tese]. São Paulo: Universidade de São Paulo, Escola de Educação Física e Esporte; 2013.

116. Rocha PGM. O efeito do controle do aprendiz sobre a auto-observação na aprendizagem motora [tese]. São Paulo: Universidade de São Paulo, Escola de Educação Física e Esporte; 2013.

117. Bastos FH. Efeito da meta de aprendizagem na aprendizagem motora autocontrolada [tese]. São Paulo: Universidade de São Paulo, Escola de Educação Física e Esporte; 2010.

118. Ferreira LF. Efeitos da intervenção aquática em crianças com características de transtorno do desenvolvimento da coordenação (TDC) [tese]. São Paulo: Universidade de São Paulo, Escola de Educação Física e Esporte; 2013.

119. Xavier Filho E. A aquisição da locomoção aquática em bebês no primeiro ano de vida [tese]. São Paulo: Universidade de São Paulo, Escola de Educação Física e Esporte; 2006.

120. Xavier Filho E. O efeito das restriçóes da tarefa e do ambiente no comportamento de locomoção no meio aquático [dissertação]. São Paulo: Universidade de São Paulo, Escola de Educação Física e Esporte; 2001.

121. Dias JABS. Desenvolvimento e aparato de suporte à locomoçáo aquática de bebês e de crianças de 3 a 24 meses [dissertação]. São Paulo: Universidade de São Paulo, Escola de Educação Física e Esporte; 2013.

122. Barbosa FM. Efeito da modificação da tarefa na braçada do nado crawl em indivíduos com níveis de habilidades distintos [dissertação]. São Paulo: Universidade de São Paulo, Escola de Educação Física e Esporte; 2006.

123. Silva CGS. Consistência e variabilidade do nado crawl em indivíduos habilidosos [dissertação]. São Paulo: Universidade de São Paulo, Escola de Educação Física e Esporte; 2008.

124. Barbosa FM. O nado de atletas de águas abertas: características do desempenho e da organização temporal das braçadas [tese]. São Paulo: Universidade de São Paulo, Escola de Educação Física e Esporte; 2016.

125. Apolinário MR. Efeitos de diferentes padrôes respiratórios no desempenho e na braçada do nado crawl [dissertação]. São Paulo: Universidade de São Paulo, Escola de Educação Física e Esporte; 2010.

126. Apolinário MR. Nado crawl: associação entre coordenação e desempenho de nadadores [tese]. São Paulo: Universidade de São Paulo, Escola de Educação Física e Esporte; 2016.

127. Cattuzzo MT. O ciclo instabilidade-estabilidade-instabilidade no processo adaptativo em aprendizagem motora [tese]. São Paulo: Universidade de São Paulo, Escola de Educação Física e Esporte; 2007.

128. Ugrinowitsch H. Efeitos do nível de estabilizaçáo do desempenho e do tipo de perturbação no processo adaptativo em aprendizagem motora [tese]. São Paulo: Universidade de São Paulo, Escola de Educação Física e Esporte; 2003.

129. Freudenheim AM. Organização hierárquica de um programa de ação e a estabilização de habilidades motoras [tese]. São Paulo: Universidade de São Paulo, Escola de Educação Física e Esporte; 1999.

130. Benda RN. Organização hierárquica de um programa de ação e a estabilização de habilidades motoras [tese]. São Paulo: Universidade de São Paulo, Escola de Educaçáo Física e Esporte; 2001. 
131. Marinovic W. Efeito da restrição da tarefa na organização do movimento de rebatida de forehand em mesa-tenistas habilidosos [dissertação]. São Paulo: Universidade de São Paulo, Escola de Educação Física e Esporte; 2003.

132. Basso L. Modularizaçáo e processo adaptativo na aquisiçáo de habilidades motoras [dissertação]. São Paulo: Universidade de São Paulo, Escola de Educação Física e Esporte; 2001.

133. Basso L. Aumento de complexidade na aprendizagem motora: efeitos dos níveis de estabilizaçáo e dos canais de desempenho [tese]. São Paulo: Universidade de São Paulo, Escola de Educação Física e Esporte; 2010.

134. Ambrósio NFA. Processo adaptativo em aprendizagem motora: um estudo centrado nas diferenças individuais do desempenho da fase de estabilização e adaptação [dissertação]. São Paulo: Universidade de São Paulo, Escola de Educação Física e Esporte; 2014.

135. Araujo UO. Liberdade na escolha da resposta e momento da estabilização em aprendizagem motora [dissertação]. São Paulo: Universidade de São Paulo, Escola de Educação Física e Esporte; 2008.

136. Bastos FH. Efeito do grau de liberdade na escolha da resposta no processo adaptativo em aprendizagem motora [dissertaçáo]. São Paulo: Universidade de São Paulo, Escola de Educação Física e Esporte; 2007.

137. Walter C. Liberdade na escolha das respostas e variabilidade de resposta no processo adaptativo em aprendizagem motora [tese]. São Paulo: Universidade de São Paulo, Escola de Educação Física e Esporte; 2014.

138. Walter C. Estrutura de prática e liberdade de escolha na aprendizagem de habilidades motoras [dissertação]. São Paulo: Universidade de São Paulo, Escola de Educação Física e Esporte; 2007.

139. Paroli R. Efeito da estrutura de prática na aquisição de uma habilidade motora [dissertação]. São Paulo: Universidade de São Paulo, Escola de Educação Física e Esporte; 2005.

140. Corrêa UC. Estrutura de prática e processo adaptativo na aquisição de habilidades motoras [tese]. São Paulo: Universidade de São Paulo, Escola de Educação Física e Esporte; 2001.

141. Pinheiro JP. A prática constante-aleatória e a diversificação de habilidades motoras [dissertação]. São Paulo: Universidade de São Paulo, Escola de Educação Física e Esporte; 2009.

142. Massigli M. Estrutura de prática e validade ecológica no processo adaptativo de aprendizagem motora [dissertação]. São Paulo: Escola de Educação Física e Esporte da Universidade de São Paulo; 2009

143. Gonçalves LA. Estrutura de prática e idade no processo adaptativo da aprendizagem de uma tarefa de timing coincidente [dissertação]. São Paulo: Universidade de São Paulo, Escola de Educação Física e Esporte; 2009.

144. Meira Junior CM. Conhecimento de resultados no processo adaptativo em aprendizagem motora [tese]. São Paulo: Universidade de São Paulo, Escola de Educação Física e Esporte; 2005.

145. Silva JAO. Estrutura de prática e complexidade da tarefa em aprendizagem motora [dissertação]. São Paulo: Universidade de São Paulo, Escola de Educação Física e Esporte; 2005.

146. Tertuliano IW. Estrutura de prática e freqüência de feedback extrínseco na aprendizagem de habilidades motoras [dissertação]. São Paulo: Universidade de São Paulo, Escola de Educação Física e Esporte; 2007.

$$
\begin{array}{r}
\text { ENDEREÇO } \\
\text { Umberto Cesar Corrêa } \\
\text { Escola de Educação Física e Esporte - USP } \\
\text { Av. Prof. Mello Moraes, } 65 \\
\text { 05508-030 - São Paulo - SP - BRASIL } \\
\text { e-mail: umbertoc@usp.br }
\end{array}
$$

110 • Rev Bras Educ Fís Esporte, (São Paulo). 2017 Ago; 31(N esp):97-110. 